\title{
Thermodynamics of extreme states of matter
}

\author{
Vladimir E. Fortova and Igor V. Lomonosov ${ }^{b}$ \\ aRussian Basic Research Foundation, 11734 Leninskii pr. 32a, Moscow, RUSSIA \\ bInstitute of Chemical Physics RAS, 142432 Chernogolovka, Moscow reg., RUSSIA
}

\begin{abstract}
Discussed is the current state of investigation of thermodynamic properties of matter at extremely high pressure and temperature with the use of dynamic methods. The generalization of available experimental and theoretical information is given in a form of the multi-phase wide-range equation of state. Topical problem of the physics of high pressures, high temperatures are discussed on the example of description of the phase diagrams for selected metals.
\end{abstract}

\section{INTRODUCTION}

Physics of high pressures, high temperatures always takes an especial place among amazing in their width scientific interests of academician N. N. Semenov. He revealed the possibilities of powerful shock waves generated by explosion for obtaining an exotic high energy concentrations under laboratory conditions immediately and in a complete volume.

N. N. Semenov conserved an instant and non-formal interest to the physics and chemistry of extreme states as well as numerous applications of the absorbing science in energetics, space, technology to his last years. He, F. I. Dubovitskii and A. N. Dremin are persons which we should acknowledge for the fact that our Institute is now a well-known center in the field of powerful shock waves and physics of extreme states of plasma.

We'll dwell in the review on one of central branches of modern science of extreme states, namely thermodynamics of hot compressed matter. The problem was discussed many times by one of the authors with Nikolai Nikolaevitch to his last years.

Given in the review are experimental and theoretical results of investigation of the phase diagrams of the metals at high pressures, high temperatures obtained, namely, by scientists from Institute of Chemical Physics in Chernogolovka Russian Academy of Sciences.

\section{POSSIBILITIES OF MODERN EXPERIMENTAL METHODS}

The current state of the problem of a theoretical description of thermodynamic properties of matter at high pressures, high temperatures is given in a set of publications (see (refs. 1-6) and references therein). In spite of a significant progress achieved on construction of equation of state (EOS) in solid, liquid and plasma state with the use of the most sophisticated "first-principle" theoretical approaches (classic and quantum methods of self-consisted field, diagram technique, computer's Monte-Carlo and molecular dynamics methods) the disadvantage of these theories is their regional character (refs. 2-6). The range of an applicability of each theory is local and, rigorously speaking, no one of them allows to provide for a correct theoretical 
calculation of thermodynamic properties of matter on the whole phase plane from the cold crystal to liquid and hot plasmas (refs. 2,3,5). The principal problem here is the necessity to take into account correctly the strong collective interparticle interaction in disordered media, which meets especial difficulties in the region occupied by dense disordered non-ideal plasmas (refs. 2-6).

In this case experimental data at high pressures, high temperatures are of peculiar significance, because they serve as reference points for theories and semiempirical models. Data obtained with the use of dynamic methods (see (refs. 1,3,4,7-10) and references therein) are of the importance from the practical point of view. Shock-wave methods allow to study a broad range of the phase diagram from compressed hot condensed phase to dense strongly coupled plasma and quasigas states. The detailed recital of shock-wave methods of investigation at high dynamic pressures is given in monographs $(1,3,4,7)$ and reviews $(8-10)$. Available experimental data on the shock compression of solid and porous metals as well as isentropic expansion embrace to nine orders with respect to pressure and four to density. Figure 1 shows on the pressure scale a modern progress achieved in measurements of an absolute shock compressibility with the use of traditional explosive technique and investigations of impedance one under conditions of experiments with concentrated energy fluxes (lasers, beams) and underground nuclear explosions.

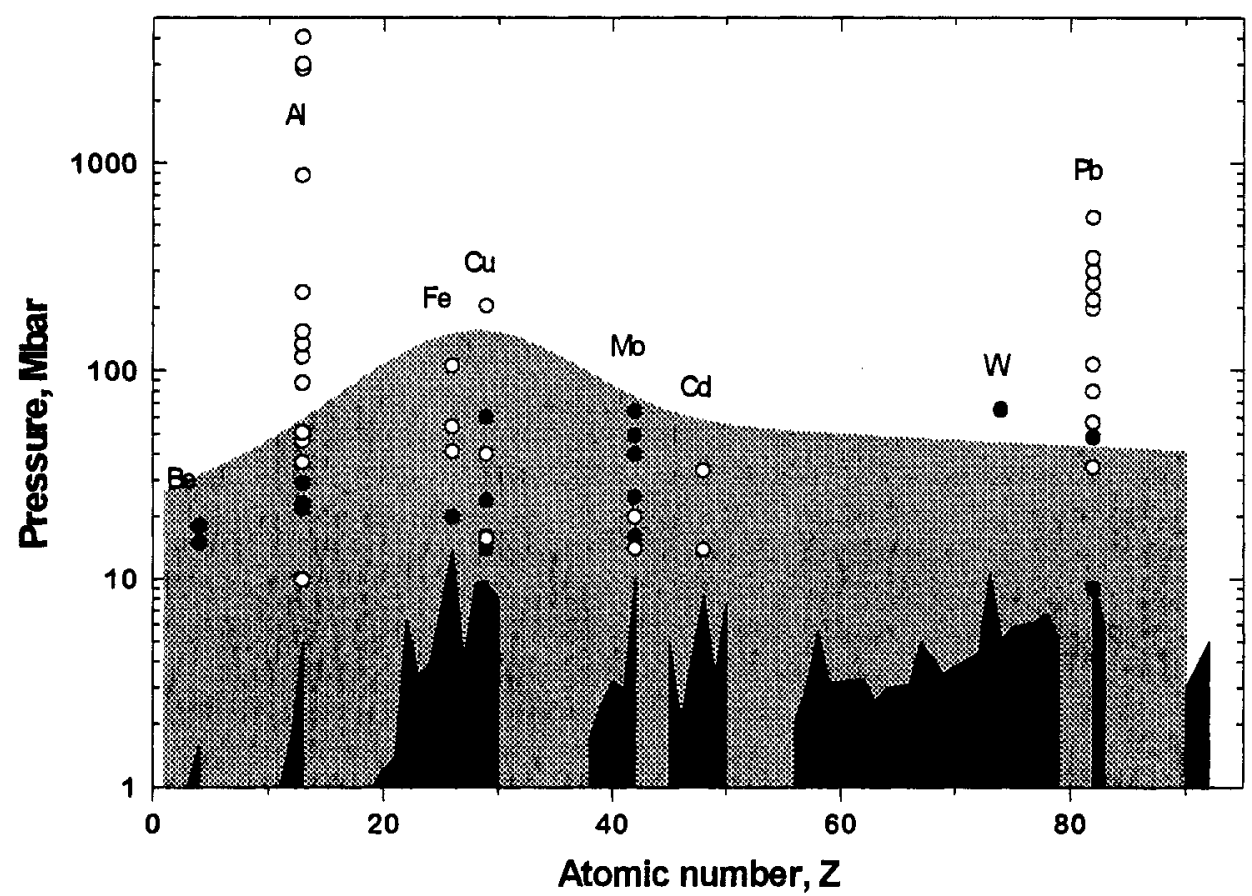

Fig. 1. The investigated pressure scale of elements. Shown are maximum pressures achieved in traditional explosive (black region), laser and beam (gray) and underground nuclear explosion (points) experiments.

The extension of studied range of the phase diagram to greater relative volumes, in comparison with the principal Hugoniot, is achieving in the investigation of the shock compressibility of porous samples (ref. 1). Nevertheless, difficulties of a work with ultradispersive targets and singularities of the heat transfer in multiphase non-equilibrium media restrict the possibility of an advance on the density scale. The method of isentropic expansion of shocked matter allows, depending on the magnitude of the shock pressure, and, respectively, the entropy provided, to pass in one experiment states from a hot metallic liquid and strongly coupled plasma to two-phase region liquid-gas and Boltzman's weakly ionized plasma and an ideal gas (refs. 1,3,4).

The complex of available experimental and theoretical information is shown on Figure 2 on 3-D relative volume-temperature-pressure surface calculated by semiempirical multi-phase EOS (ref. 4). It is well seeing 
that besides registrations of the shock compressibility, measurements of release isentropes of shocked materials are of especial importance. Such results allow to pervade in the intermediate between solid state and gas region occupied by a dense hot metallic liquid and strongly coupled plasma (refs, 3,4 ), which is very difficult for theory. Experimentally studied release isentropes for copper has as initial high energy states of solid, melted and compressed liquid metal. The range of thermodynamic parameters covered in the adiabatic expansion process for these states is extremely wide (Fig. 2), five orders of magnitude with respect to pressure and two orders of magnitude with respect to density. It extends from a highly compressed metallic liquid characterized by a disordered arrangement of ions and degenerate electrons to a quasi-non-ideal Boltzmann plasma and a rarefied metallic vapor. Upon expansion of the system the degree of degeneracy of the electronic subsystem is decreased and a marked rearrangement of the energy spectrum of atoms and ions occurs, and a partial recombination of the dense plasma takes place. In the disordered electron system a "metal-insulator" transition takes place and a non-ideal (with respect to different forms of interparticle interactions) plasma is formed in the vicinity of the liquid-vapor equilibrium curve and the critical point. Where the isentropes enter the two-phase liquid-vapor region evaporation occurs; on the gas-side condensation occurs (refs. 3,4,8).

Note, that typical shock-wave measurements allow to determine only caloric properties of matter, viz. the dependence of the relative internal energy on pressure and volume as $E=E(p, V)$. The potential $E(p, V)$ is not complete in the thermodynamic sense and a knowledge of temperature $T$ or entropy $S$ is required for completing thermodynamic equations and calculating first and second derivatives, such as the heat capacity, the sound velocity and others (ref. 3 ).

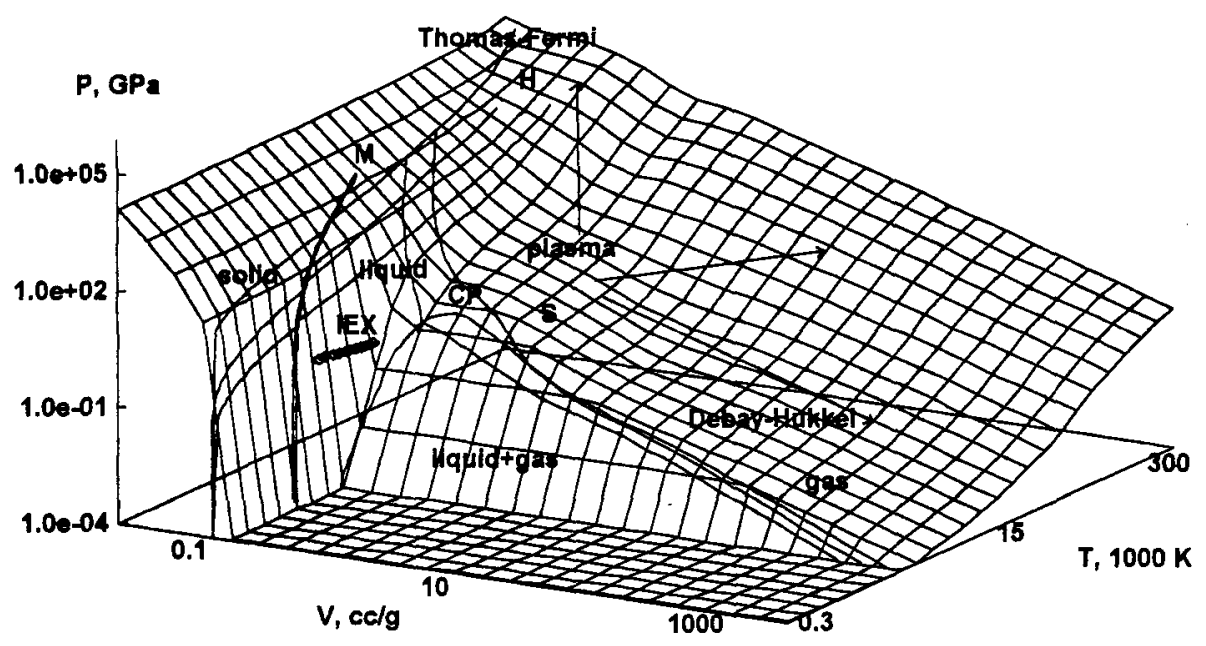

Fig. 2. 3-D volume-temperature-pressure surface for copper in the investigated region of the phase diagram. M - melting region, $\mathrm{H}$ - principal and porous Hugoniots, IEX - isobaric expansion data, S - release isentropes, CP - the critical point. Shown are phase states of metal; arrows indicate the direction of the nonideality decreasing.

Only few temperature registrations in shocked metals are available (ref. 11), as well as analogous measurements in release isentropic waves (ref. 8). Meanwhile this information is of great importance in view of a limitation of pure theoretical calculation methods. From this point of view thermodynamically complete measurements obtained with the use of isobaric expansion (IEX) technique (ref. 12) are of a special significance. In this method metal is rapidly heated by a powerful pulsed current and then it expands into an atmosphere of the inertial gas maintaining at constant pressure. These data embrace the density to critical one and intersect, therefore, the release isentrope data for metals (see Figure 2). 
The final conclusion is that the available at high pressures, high temperatures information though covers a broad range of the phase diagram, but it has a heterogeneous character and, as a rule, is not complete from thermodynamic point of view. Its generalization can be done only in form of thermodynamically complete EOS. Used as such EOS was one of the most advanced multi-phase EOS for metals proposed by A. V. Bushman and developed in (ref. 4).

\section{MULTI-PHASE WIDE-RANGE EOS MODEL}

A thermodynamically complete temperature EOS for metal is defined by the thermodynamic potential of free energy, F, written as,

$$
F(V, T)=F_{c}(V)+F_{a}(V, T)+F_{e}(V, T)
$$

describing the elastic contribution at $\mathrm{T}=0 \mathrm{~K}\left(\mathrm{~F}_{\mathrm{c}}\right)$, and the heat contribution by atoms $\left(\mathrm{F}_{\mathrm{a}}\right)$ and electrons $\left(\mathrm{F}_{\mathrm{e}}\right)$, respectively.

\section{Cold curve}

The elastic energy for the solid phase is given in form

$$
F_{c}^{(s)}(V)=3 V_{0 c} \sum_{i=1,5} \frac{a_{i}}{i}\left(\sigma_{c}^{i / 3}-1\right)
$$

(where $\sigma_{c}=V_{0 c} / V, V_{0 c}$ - specific volume at $p=0$ ) providing for conditions $F_{c}^{(s)}\left(V_{0 c}\right)=0, p_{c}\left(V_{0 c}\right)=0$, correct tabular values (with heat component) of bulk compression modulus and its pressure derivative at ambient conditions, as well as the accordance with first-principle theories to 100 -fold compression (ref. 2).

The cold energy for the liquid phase in compression region $\left(\sigma_{c} \geq 1\right)$ is given by Eq. (2), while in the rarefaction region $\left(\sigma_{c}<1\right)$ it is represented by a polynomial of the form

$$
F_{c}^{(1)}(V, T)=V_{0 c}\left[A\left(\sigma_{c}^{m} / m-\sigma_{c}^{n} / n\right)+B\left(\sigma_{c}^{1} / 1-\sigma_{c}^{n} / n\right)\right]+E_{\text {sub }}
$$

which leads to the tabular values of the cohesion energy $E_{\text {sub }}$ and satisfies the condition $p_{c}\left(V_{0 c}\right)=0$. The equality of the cold-energy derivatives $p_{c}=-d E_{c} / d V, B_{c}=-d p_{c} / V d V, B_{p c}=d B_{c} / d p$ at $\sigma_{c}=1$ remains in (3) two free parameters, $n$ and 1 . They are determined by the experimental temperature dependence of density and sound velocity for liquid metals.

\section{Thermal contribution of the atoms in the solid state}

The lattice contribution to the free energy of the solid phase is defined by the expression

$$
F_{a}^{(s)}(V, T)=3 R T \ln \frac{\theta_{c}^{(s)}(V)}{T}
$$

where $\mathbf{R}$ is the gas constant. The characteristic temperature is given by empirical expression

$$
\theta_{c}^{(s)}(V)=\theta_{0}^{s} \sigma^{2 / 3} \exp \left\{\frac{\left(\gamma_{0 s}-2 / 3\right)\left(B_{s}^{2}+D_{s}^{2}\right)}{B_{s}} \operatorname{arctg}\left[\frac{x B_{s}}{B_{s}^{2}+D_{s}\left(x+D_{s}\right)}\right]\right\},
$$

where $x=\ln \sigma$. Constants $B_{s}$ and $D_{s}$ are determined by the experimental dependence of Gruneisen gamma $\gamma=\mathrm{d} \ln \theta / \mathrm{d} \ln \sigma$ on compression $\sigma$. This dependence is obtained on the base of shock-wave data. The value of $\gamma_{0 s}$ corresponds to tabular one of Gruneisen gamma at ambient conditions. Note that at high compression 
Eq. (5) provides for the correct ideal-gas asymptote $\cong \sigma^{2 / 3}$. The value of $\theta_{0}^{\mathrm{s}}$ is given by condition $\mathrm{s}\left(\mathrm{V}_{0}\right)=0$.

\section{Thermal contribution of the atoms in the liquid state}

The atomic contribution to the free energy of the liquid phase is represented as

$$
F_{a}^{(l)}(V, T)=F_{t}(V, T)+F_{m}(V, T) ;
$$

the first term accounts for anharmonicity effects and the second provides for a proper behavior of melting curve.

In the liquid phase the phonon contribution has a form similar to Eq. (4) but with a volume- and temperature-dependent heat capacity $c_{\mathbf{a}}$ and a characteristic temperature $\theta(\mathrm{l})$

$$
F_{t}^{(1)}(V, T)=c_{a}(V, T) T \ln \frac{\theta^{(1)}(V, T)}{T}
$$

The heat capacity in the liquid phase is given by the expression

$$
c_{a}(V, T)=\frac{3 R}{2}\left[1+\frac{\sigma T_{a}}{\left(\sigma+\sigma_{a}\right)\left(T+T_{a}\right)}\right],
$$

describing a smooth variation from the value $3 R$ close to the lattice heat capacity to that one of an ideal atomic gas, $3 R / 2$. Coefficients $\sigma_{a}$ and $T_{a}$ define the characteristic density and temperature of this transition.

The vibrational spectrum defined by the variation of the characteristic temperature reflects the gradual change of the Gruneisen coefficient of the liquid phase from values $\gamma^{(1)} \approx \gamma^{(s)}$ corresponding to condensed states to the ideal-gas value of $2 / 3$ in the limit of high temperatures and very low densities. Under these assumptions, the characteristic temperature is given by the approximating formula

$$
\theta^{(1)}(V, T)=T_{s a} \frac{\left(T_{c a} \theta_{c}^{(1)}+T\right) \sigma_{c}^{2 / 3}}{T_{c a}+T}
$$

where characteristic temperature in liquid phase is given in form analogous to Eq. (5)

$$
\theta_{c}^{(1)}(V)=\theta_{0}^{1} \exp \left\{\frac{\left(\gamma_{01}-2 / 3\right)\left(B_{1}^{2}+D_{1}^{2}\right)}{B_{1}} \operatorname{arctg}\left[\frac{x_{1}}{B_{1}^{2}+D_{1}\left(x+D_{1}\right)}\right]\right\} \text {. }
$$

The parameters in Eq. (10) $B_{1}$ and $D_{1}$ are found from shock-wave experiments on solid and porous samples, the constant $\theta_{0}^{1}$ is determined by equation $\theta_{\mathrm{a}}(0)=\mathrm{T}_{\mathrm{ca}}$.

The potential term $F_{m}(V, T)$ provides for a correct value of the entropy $\Delta s=\Delta s_{m 0}$ and volume $\Delta \mathrm{V}=\Delta \mathrm{V}_{\mathrm{m} 0}$ changes on melting at ambient pressure and disappears in the gas phase. The contribution of $F_{m}$ should also decrease upon compression due to decreasing differences between the properties of the solid and liquid phases. These requirements are satisfied by the relation

$$
F_{m}(V, T)=3 R\left\{\frac{2 \sigma_{m}^{2} T_{m 0}}{1+\sigma_{m}^{3}}\left[C_{m}+\frac{2 A_{m}}{5}\left(\sigma_{m}^{5 / 3}-1\right)\right]+\left(B_{m}-C_{m}\right) T\right\},
$$

where $\sigma_{m}=\sigma / \sigma_{m 0}$ is the relative density of the liquid phase on the melting curve. The constants $A_{m}, B_{m}, C_{m}$ are uniquely determined by the equilibrium conditions along the melting curve at $T=T_{m}$. 


\section{Thermal contribution of electrons}

The thermal electron's contribution has the same form for solid and liquid phases. It is given by following relationship

$$
F_{e}(V, T)=-c_{e}(V, T) T \times \ln \left[1+\frac{B_{e}(T) T}{2 c_{e i}} \exp \left(-\gamma_{e}(V, T)\right)\right]
$$

It includes the generalized analog of the coefficient of electron heat capacity $B_{e}$, the heat capacity of the electron gas $c_{e i}$, the analogs of the electronic Gruneisen coefficient $\gamma_{e}$, the coefficient of the electronic heat capacity $\beta_{\mathrm{e}}$ :

$$
\begin{aligned}
& B_{e}(T)=\frac{2}{T^{2}} \int\left(\int_{0}^{T} \beta(\tau) d \tau\right) d T, \beta(T)=\beta_{i}+\left(\beta_{0}-\beta_{i}+\beta_{m} \frac{T}{T_{b}}\right) \exp \left[-\frac{T}{T_{b}}\right], \\
& c_{e i}(V, T)=\frac{3 R}{2}\left[Z+\frac{\sigma_{z} T_{z}^{2}(1-Z)}{\left(\sigma+\sigma_{z}\right)\left(T^{2}+T_{z}^{2}\right)}\right] \exp \left[-\frac{\tau_{i}}{T}\right], \quad \tau_{i}=T_{i} \exp \left(-\sigma_{i} / \sigma\right), \\
& \gamma_{e}(V, T)=\gamma_{e i}+\left(\gamma_{e 0}-\gamma_{e i}+\gamma_{m} T / T_{g}\right) \exp \left[-T / T_{g}-\left(\sigma-\sigma_{e}\right)^{2} / \sigma \sigma_{d}\right]
\end{aligned}
$$

Approximating dependencies are selected such to satisfy primarily the asymptotic relations for the electron gas free energy, namely expressions for degenerate electron gas $F_{e}(V, T)=-\sigma^{-\gamma} 0 \beta_{0} T^{2} / 2$ at moderate $\left(\mathrm{T}<\mathrm{T}_{\text {Fermi }}\right)$ temperatures and expressions for an ideal electron gas at $\mathrm{T} \rightarrow \infty$ range $F_{e}(V, T)=3 R Z \ln \left(\sigma^{2 / 3} T\right) / 2$. Here $Z$ is the atomic number and $R$ is the gas constant. The specific form of writing down separate expressions of the potential (12) depends upon the satisfaction of these requirements.

The Eqs. (12)-(17) are written in a form which represents correctly the primary ionization effects in the plasma region and the behavior of the partially ionized metal. The expression (16) for $\tau_{\mathrm{i}}$ describes a decrease of the ionization potential as the plasma density increases, and the constants $\sigma_{z}$ and $T_{z}$ define, respectively, the characteristic density of the 'metal-insulator' transition and the temperature dependence of the transition from a singly ionized gas to a plasma with the ion-charge mean value $Z$.

\section{PROCEDURE OF CONSTRUCTING EOS}

The set of equations (1)-(17) fully assigns the thermodynamic potential for metals over entire phase diagram region of practical interest. Some coefficients in EOS, included in the analytical expressions, are constants characteristic for each metal (atomic weight and charge, density at normal conditions and other) and are found from tabular data, while the rest serve as fitting parameters and their values are found from the optimum description for the available experimental and theoretical data and providing for correct asymptotes to calculations on the base of Debay-Hukkel and Thomas-Fermi theories (ref. 15). It should be emphasized that, even though the number of coefficients in Eqs. (1)-(17) is large, most of them are rigidly defined constants whose values are assigned explicitly or implicitly from the fulfillment of various thermodynamic conditions at specific points on the phase diagram. A few coefficients (about ten) serve to characterize the densities and temperatures on transition from one typical phase-plane region to another and are found empirically.

In determining the numerical values of coefficients in the EOS, use was made of the numerous experimental and theoretical data characterizing the thermodynamic properties of metals under study for a wide range of parameters. This procedure was carried out with the aid of a specially developed computer program using the Eqs. (1)-(17) for thermodynamic calculations. At the preliminary stage of calculations, some thermodynamic constants known for each substance (such as normal density; changes in density and entropy at the melting point under normal pressure; cohesion energy; and the like) were used by the program 
automatically find a number of other uniquely defined coefficients (parameters of the cold curve, melting curve, and so on). This further enables one, by way of calculating algebraic or integral relations valid for self-similar hydrodynamic flows to perform calculations of kinematic characteristics measured experimentally at high and ultrahigh pressures, namely, the incident and reflected wave velocities and velocities in adiabatic expansion waves (release isentropes), as well as to allow for melting and evaporation effects in the results obtained. The range of action of each fitting coefficient that remains free is very localized, as a result of which its value may be selected independently from a comparison of the calculations with available experimental data.

To construct EOS, used was following information at high pressures, high temperatures: measurements of isothermal compressibility in diamond anvil cells, data on sound velocity and density in liquid metals at atmospheric pressure, IEX measurements, registration of shock compressibility for solid and porous samples in incident and reflected shock waves, impedance measurements of a shock compressibility under condition of an underground nuclear explosion, data on isentropic expansion of shocked metals, calculations by Debay-Hukkel and Thomas-Fermi models, evaluations of the critical point.

\section{THERMODYNAMIC PROPERTIES OF NICKEL AND LEAD}

Engineering calculations and involving of the real thermodynamic information into gasdynamic codes for numerical modeling of physical processes, occurring under conditions of extremely high pressures and temperatures, are final goals of construction EOS. The accuracy and reliability of such calculations depend on, namely, EOS used. This is the reason why on developing EOS for nickel and lead a special attention was paid on the accuracy and reliability of description of not only caloric, but also temperature data as well as numerous static measurements at high pressure.

\section{Nickel}

As it is seen from Figure 3, the obtained for nickel cold curve agrees with calculations on the base of Thomas-Fermi model with quantum and exchange corrections (ref. 15) to 100 -fold compressions. It also provides for good agreement with augmented plane wave's calculations (ref. 16) and results of early semiempirical models.

According to (ref. 17), nickel remains in fcc-phase to pressure of $65 \mathrm{GPa}$. The calculated value of the slope of the melting curve $\mathrm{dT} / \mathrm{dP}=4.4 \mathrm{~K} / \mathrm{kbar}$ is in a satisfactory agreement with a value $3.3 \mathrm{~K} / \mathrm{kbar}$ cited in compendium (ref. 18) (determined to pressure $60 \mathrm{kbar}$ ).

The principal Hugoniot of nickel has been studied by virtue of traditional shock-wave generators to 1.5 Mbar in works (refs. 19-21) and light-gas gun to $5 \mathrm{Mbar}$ (ref. 22). Usage of special powerful high-explosive systems increased the limit of investigated pressure to ca. $10 \mathrm{Mbar}$ (refs. 23,24). Data on shock compression of porous (refs. 23,25) and ultra porous (ref. 26) nickel samples in a megabar-pressure range significantly expanded the region of the investigated phase diagram. Results of work (ref. 26) are of especial interest as they occupy the region of strongly heated liquid metal and, for the most porous shock Hugoniot $\mathrm{m}=\rho_{0} / \rho_{00}=20$, correspond to the state of weakly ionized nonideal $(\Gamma=0.1-0.3)$ plasma.

The calculated phase diagram of nickel is shown on Figure 4 in comparison with available shock-wave data. Note, that results (ref. 23) for porosity $m=1.73$ do not agree with those one from later work (ref. 25), while for $m=3.0$ their position on the phase diagram corresponds to the position of porous shock Hugoniots $\mathrm{m}=4.58,2.72,2.32$ from (ref. 25). The analysis of experimental data on shock compression of porous specimens shows, that the position of porous and ultra porous Hugoniots is reasonable (see Fig. 4). The principal Hugoniot data for nickel, obtained with the use of different shock-wave generators, are also in a good agreement with each other. This unique set of data embraces the region of density $0.2<\rho / \rho_{0}<2$ to pressure ca. $10 \mathrm{Mbar}$ and gives an opportunity to account for precisely thermal contribution of electrons and ions in EOS. Calculated shock adiabats of different initial densities describe with very high accuracy all available shock-wave data for nickel. 


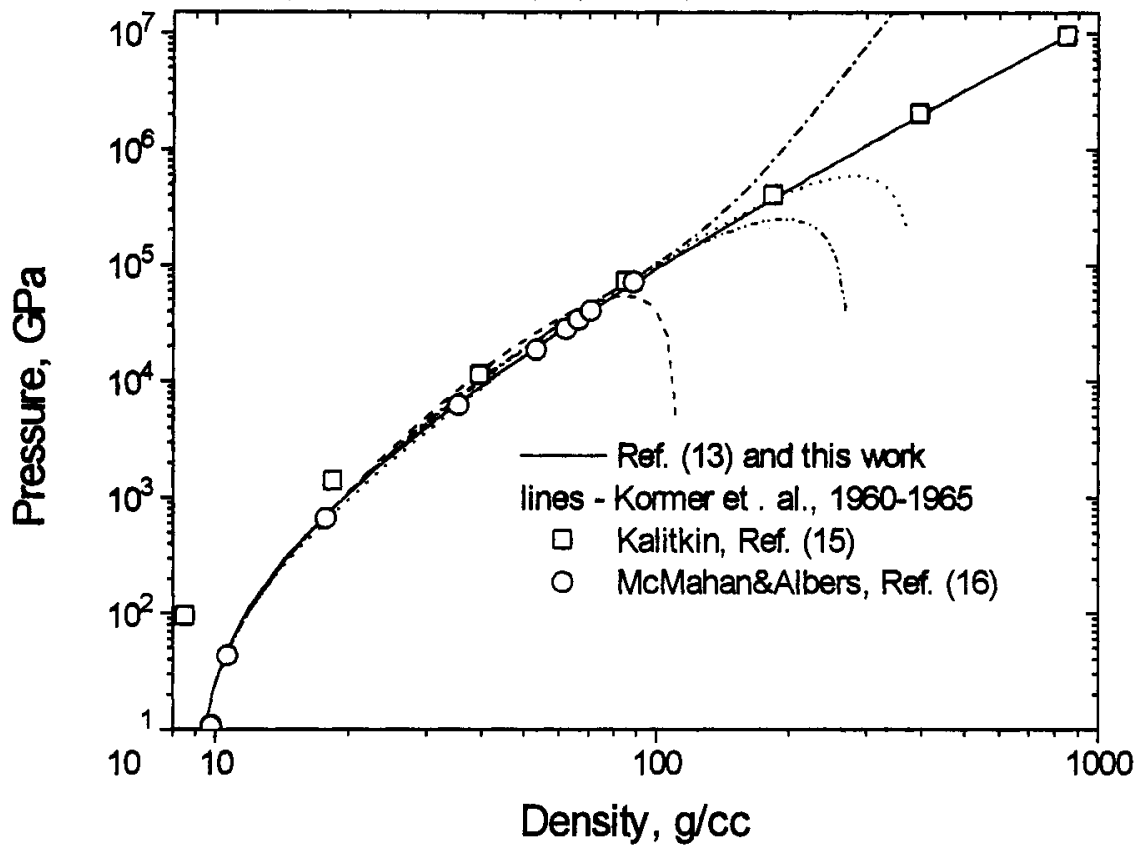

Fig. 3. Pressure in nickel at $\mathrm{T}=0 \mathrm{~K}$.

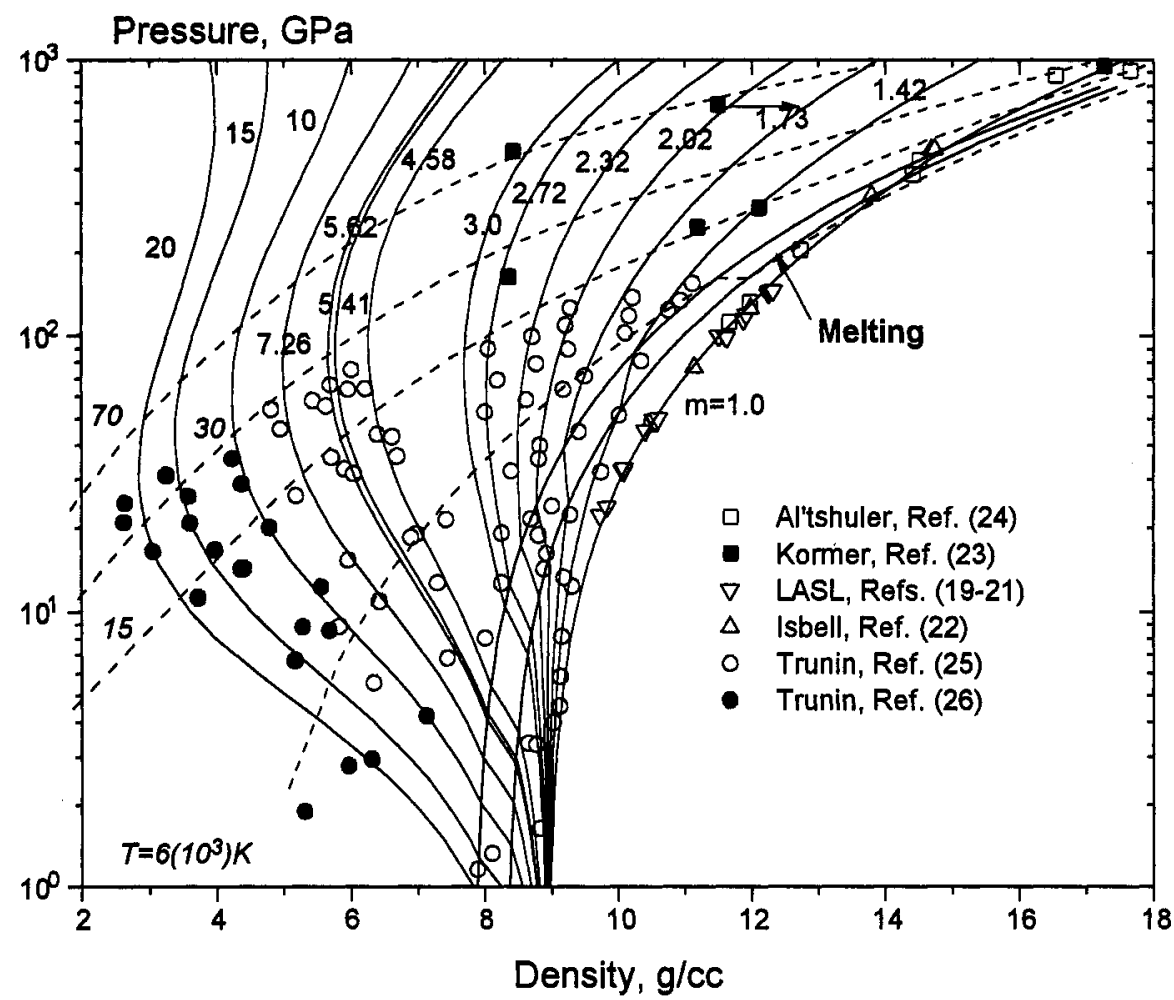

Fig. 4. The phase diagram of nickel at high pressure. $\mathrm{m}$ - Hugoniots of porous samples, $\mathrm{T}$ - isotherms, points - experiment. 


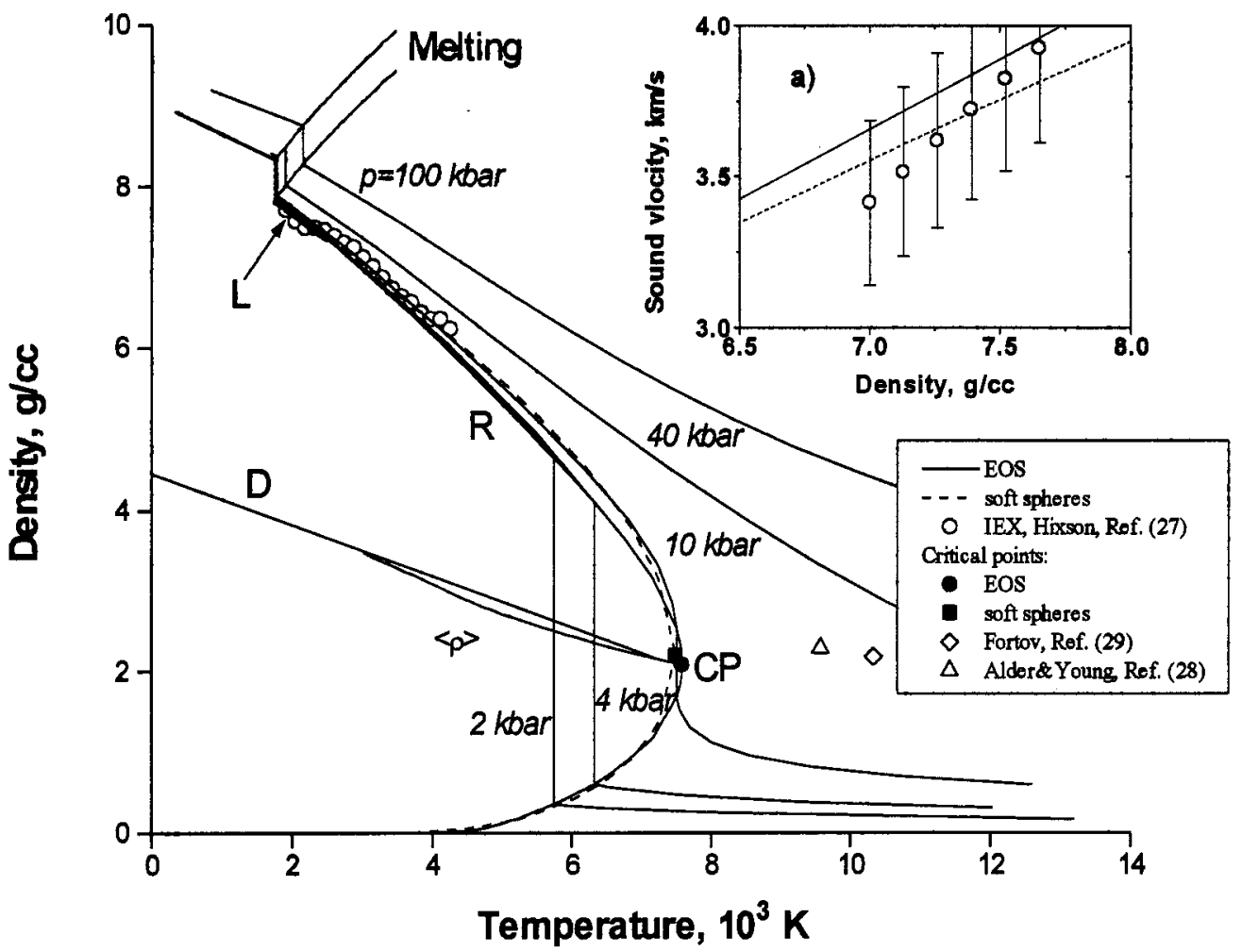

Fig. 5. The phase diagram for nickel in the region of lower density. $M$ - melting region, $R$ - boundary of liquid-vapor region with the critical point $\mathrm{CP}, \mathrm{L}$ - density of liquid nickel at $\mathrm{p}=1$ bar, $\mathrm{D}-$ law of rectilinear diameter, $\langle\rho\rangle-$ half sum of density of liquid and gas, $\mathrm{p}$ - isobars, points - experiment and theory. a) Sound velocity in liquid nickel at $\mathrm{p}=3 \mathrm{kbar}$.

Analogous to shock-wave data, there is a good correspondence for nickel between all available experimental data and theoretical predictions in the region of lower densities and moderate temperatures, as it is demonstrated on Figure 5. The nickel EOS describes with very good accuracy results of experimental work (ref. 27). Calculated parameters of the critical point $p_{c}=10.49 \mathrm{kbar}, T_{c}=7585 \mathrm{~K}, V_{c}=0.479 \mathrm{cc} / \mathrm{g}$ and $\mathrm{s}_{\mathrm{c}}=2.526 \mathrm{~J} / \mathrm{gK}$ are in agreement with available evaluations (refs. 28,29). Note that the value of the Ni critical temperature from EOS is less then that from (refs. 28,29). It is reasonable as for tantalum, for instance, the value of critical temperature has been changed from $17330 \mathrm{~K}$ (ref. 28) to $9284 \mathrm{~K}$ (ref. 30), when the hardspheres model is substituted for soft-spheres one. The value of evaporation temperature at normal pressure $\mathrm{T}_{\mathrm{v}}=3020 \mathrm{~K}$ agrees with the tabular one $3190 \mathrm{~K}$ from (ref. 14) (note, that it can differ from other references in $\pm 10 \%$ ).

Nickel is one of the most investigated metals. Both theoretical and experimental high-pressure data for nickel are in an excellent agreement in a broad range of density and pressure.

Lead

The detailed comparison between calculated and experimental shock Hugoniots, release isentropes, results of static measurements for lead is given in monograph (ref. 4). So an analysis of the phase diagram for lead, which takes into account recent temperature data obtained in isentropic expansion waves (ref. 31), is of interest. A correction of EOS parameters for lead has been done, which does not change significantly the description of information given in (ref. 4). 


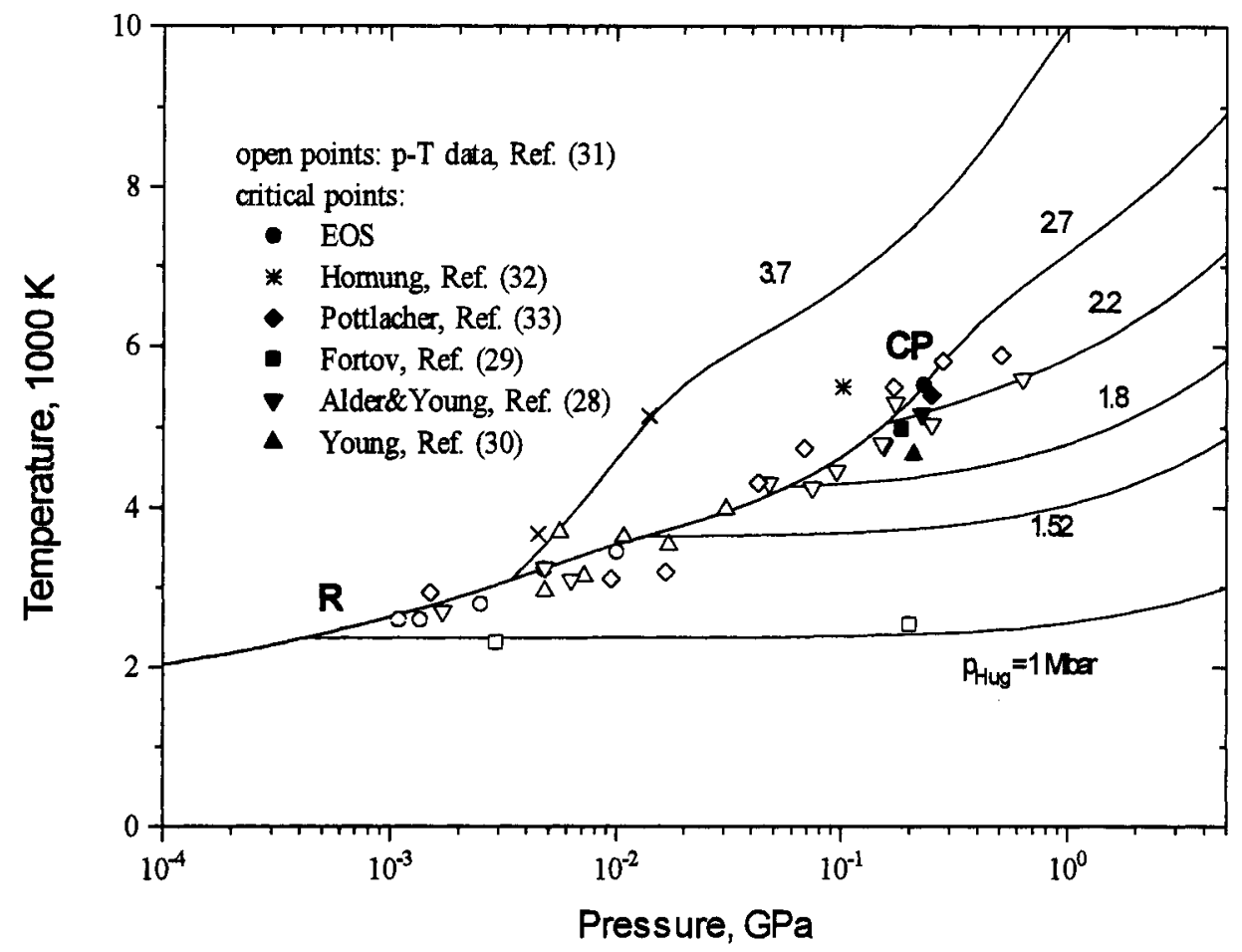

Fig. 6. The phase diagram for lead near evaporation region. $R$ - calculated evaporated region with the critical point $C P, P_{H u g}-$ shock pressure on the release isentrope.

Figure 6 illustrates the comparison between calculated and experimental release isentropes of lead (ref. 31) in the region of evaporation and in the vicinity of the critical point. As it is seen fro Figure 6 , these temperature registrations on the release isentropes allow one to determine experimentally the position of the evaporation curve over the whole range of temperature up to the critical one. The good accordance of measurements belonging to different values of initial shock pressure, and, therefore, position of entry of isentropes into two-phase region liquid-vapor both from liquid and plasma site, proves the fact. Modified EOS for lead agrees very good with temperature measurements (ref. 31) and evaluations of the critical point (refs. 28-30,32,33).

The comparison with IEX data (ref. 34) is shown on calculated phase diagram of lead in the region of lower densities on Figure 7. The developed EOS, as it is seen from Figure 7, describes very good results (ref. 34), shows correctly the thin effect of curvature of the experimental isobar when it passes near the critical point, and agrees with all available estimations of the critical point.

As consequence, let's apply procedure proposed in (ref. 1) for determination the shock temperature using the experimental dependence of pressure upon expansion velocity $p(u)$ in release isentrope wave and IEX data. Combining the simplest approximation for $p(u)$ from experiment (ref. 35) with Rieman's invariant for the self-similar flow, one can easily obtain on the isentrope $E=E(p, V)$. The intersection of isentrope with IEX data (ref. 34) gives the temperature on the isentrope, and, following the idea (ref. 1), allows one to determine the value of the shock temperature. Obtained in this manner values for temperature in shocked lead: $5100 \mathrm{~K}$ at $\mathrm{p}=920 \mathrm{kbar}$ and $9900 \mathrm{~K}$ at $\mathrm{p}=1.47 \mathrm{Mbar}$ are in reasonable agreement with experimental data and theoretical evaluations. 


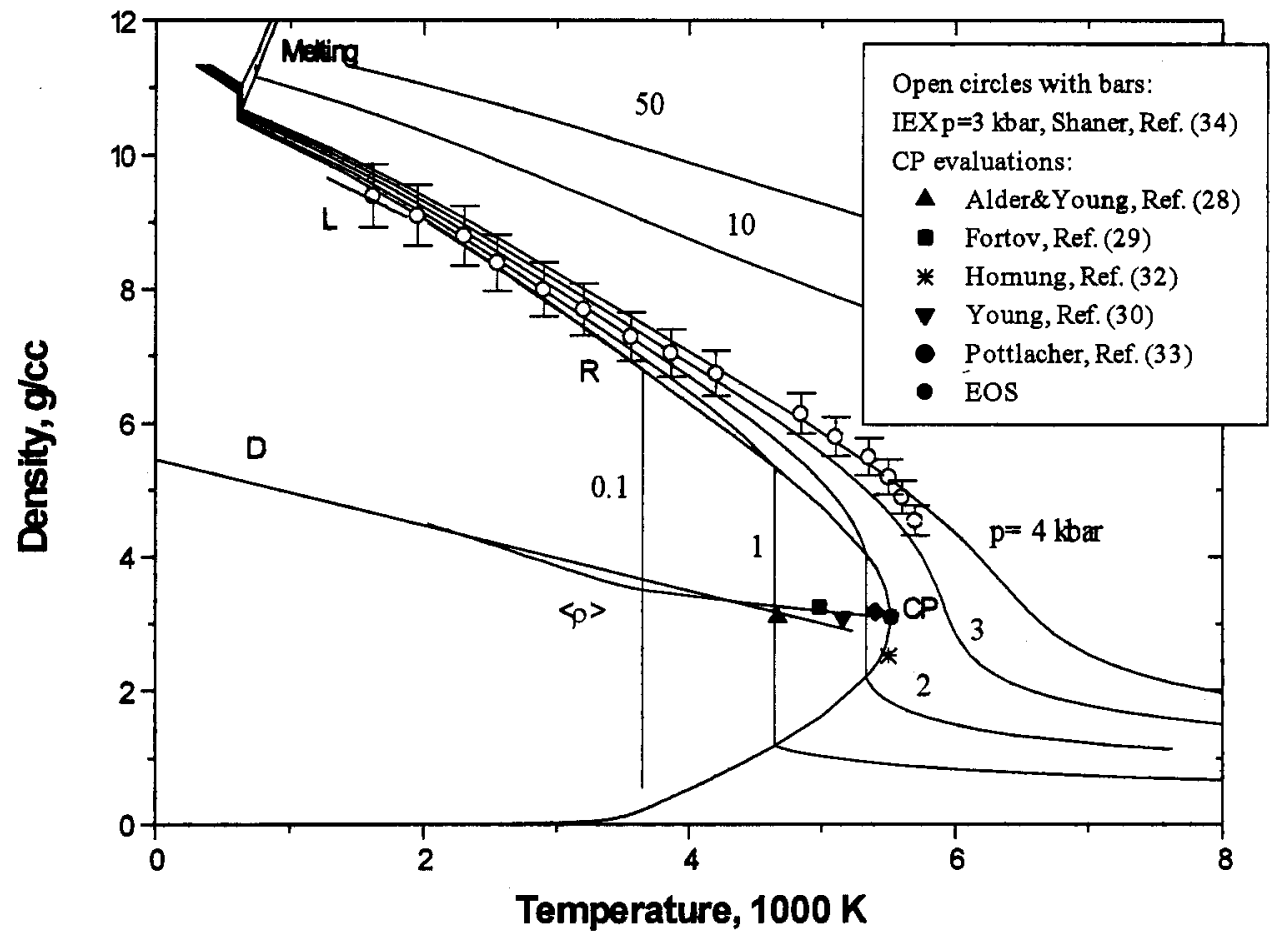

Fig. 7. The phase diagram for lead in the region of lower density. Captions are the same as on Fig. 5.

\section{CONCLUSION}

Results of analysis of the phase diagrams done for numerous simple and refractory metals show that the multi-phase wide-range EOS describes in consistent manner the whole complex of nonuniform theoretical and experimental information at high pressures, high temperatures. It is proved, that caloric measurements of shock Hugoniots and release isentropes agree with thermodynamically complete data. So, the information obtained in shock-wave experiment, which is gas dynamic in its origin, and results of temperature registrations are self consistent.

We see, that the recent progress achieved in the experimental study of extreme states by use of shock-wave methods made it possible not only generate exotic states of a hot compressed matter, but also realize the physically noncontradictory description of their thermodynamic properties in the whole region of the phase diagram, which is interesting for modern applications.

\section{REFERENCES}

1. Ya. B. Zeldovich and Yu. P. Raizer. Physics of Shock Waves and High-Temperature Hydrodynamic Phenomena. Academic Press, New York $(1966,1967)$.

2. S. Elieser, A. K. Ghatak, H. Hora. An Introduction to Equations of State: Theory and Applications. Cambridge Univ. Press, Cambridge (1986).

3. V. E. Fortov and I. T. Yakubov. Non-ideal plasma. Atomenergoizdat, Moskva (1994) [in Russian].

4. A. V. Bushman, G. I. Kanel, A. L. Ni, V. E. Fortov. Intense Dynamic Loading of Condensed Matter. Taylor\&Francis, London (1993).

5. A. V. Bushman and V. E. Fortov. Sov. Phys. Usp., 26, 465 (1983).

6. M. Ross. Rep. Progr. Phys. 48, 1-52 (1985).

7. R. G. McQueen, S. P. Marsh, J. W. Taylor, J. N. Fritz, W. J. Carter. In High Velocity Impact Phenomena (R.Kinslow, ed.), p.293-417; appendies on pp. 515-568. Academic Press, New-York. (1970) 
8. E. N. Avrorin, B. K. Vodolaga, V. A. Simonenko, V. E. Fortov. Powerful Shock Waves and Extreme States of Matter. İ.: IVTAN, Moskva (1990) [in Russian].

9. L. V. Al'tshuler. Sov. Phys. Usp. 8, 52 (1965).

10. G. E. Duvall, R. A. Graham. Rev. Modern Phys. 49, 523 (1977).

11. C. S. Yoo, N. C. Holmes, M. Ross, D. J. Webb, C. Pike. Phys. Rev. Lett. 70(25), 3931 (1993).

12. G. R. Gathers. Rep. Progr. Phys. 49, 341 (1986).

13. A. V. Bushman, I. V. Lomonosov, V. E. Fortov. Equations of State for Metals at High Energy Density. Inst. Chem. Phys., Chernogolovka (1992) [in Russian].

14. R. Hultgren, P. D. Desai, D. T. Hawkins, M. Gleiser, K. K. Kelley, D. D. Wagman. Selected Values of the Thermodynamic Properties of the Elements. ASME, Metals Park, Ohio (1973).

15. N. N. Kalitkin, L. V. Kuz'mina. Reprint N35 Inst. Prikl. Matem. Akad. Nauk SSSR, Moskva (1975) [in Russian].

16. A. K. McMahan, R. C. Albers. Phys. Rev. Lett. 49, 1198 (1982).

17. D.A. Young. Phase Diagrams of the Elements. Univ. of California Press, Berkeley (1991).

18. E. Yu. Tonkov. Phase Diagrams of Elements at High Pressure. Nauka, Moscow (1977) [in Russian].

19. J. M. Walsh, M. H. Rice, R. G. MoQueen, F. L. Yarger. Phys. Rev., 108, 196 (1957).

20. R. G. McQueen, S. P. Marsh. J. Appl. Phys. 31, 1253 (1960).

21. LASL Shock Hugoniot Data (S. P. Marsh, ed.). Univ. of California Press, Berkeley (1980).

22. W. H. Isbell, F. H. Shipman, A. H. Jones. General Motors Corp.: Mat. Sci, Lab. Report MSL-68-13 (1968).

23. S. B. Kormer, A. I. Funtikov, V. D. Urlin, A. N. Kolesnikova. Sov. Phys. - JETP 15, $477-478$ (1962).

24. L. V. Al'tshuler, A. A. Bakanova, R. F. Trunin. Sov. Phys. -JETP 15, 65 (1962).

25. R. F. Trunin, G. V. Simakov, Yu. N. Sutulov, A. B. Medvedev, B. D. Rogozkin, Yu. E. Fedorov. Sov. Phys. - JETP 69, 580 (1989).

26. R. F. Trunin, G. V. Simakov. Sov. Phys. - JETP 76, 1090 (1993).

27. R. S. Hixson, M. A. Winkler, M. L. Hodgson Phys. Rev. Ser. B 32, 6485 (1990).

28. D. A. Young, B. J. Alder. Phys. Rev. Ser. A 3, 364 (1971).

29. V. E. Fortov, A. N. Dremin, A. A. Leont'ev. Teplofiz. Visokh. Temper. 13, 1072 (1975) [in Russian].

30. D. A. Young. UCRL-52352 (Lawrence Livermore Laboratory, 1977).

31. V. Ya. Ternovoi, V. E. Fortov, S. V. Kvitov, D. N. Nikolaev. In Proceedings of conference "Shock Waves in Condensed Matter - 1995" (to be published).

32. K. Hornung. J. Appl. Phys. 46, 2548 (1975).

33. G. Pottlacher, H. Jager. Int. J. Thermophys. 11(4), 719 (1990).

34. J. W. Shaner, G. R. Gathers, W. M. Hogson. In Proceed. of Seventh Symp. on Thermophys. Properties. ASME, New York, pp.896-903 (1977).

35. M. V. Zhernokletov, G. V. Simakov, Yu. N. Sutulov, R. F. Trunin. Teplofiz. Visok. Temper. 33, 40 (1995) [in Russian] 